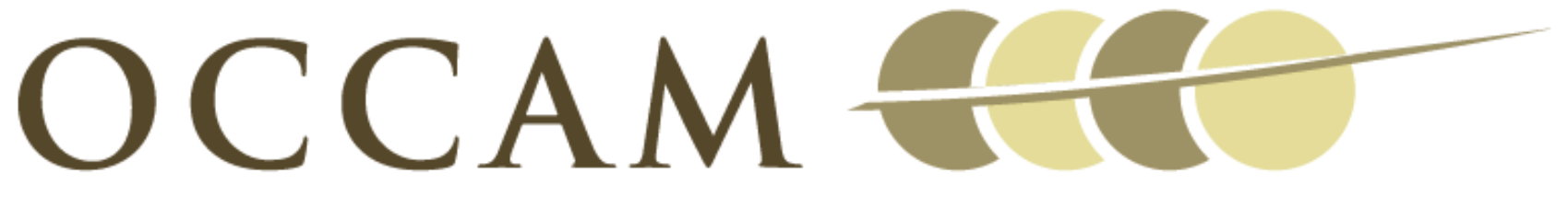

OXFORD CENTRE FOR COLLABORATIVE APPLIED MATHEMATICS

Report Number 11/47

Effects of demographic noise on the synchronization of a metapopulation in a fluctuating environment

by

Yi Ming Lai, Jay Newby, and Paul C. Bressloff

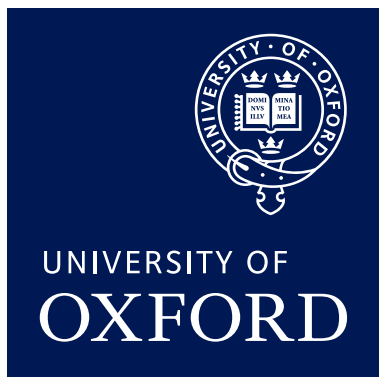

Oxford Centre for Collaborative Applied Mathematics Mathematical Institute 24 - 29 St Giles'

Oxford

OX1 3LB

England 



\title{
Effects of demographic noise on the synchronization of a metapopulation in a fluctuating environment
}

\author{
Yi Ming Lai ${ }^{1}$, Jay Newby ${ }^{1}$ and Paul C. Bressloff ${ }^{1,2}$ \\ ${ }^{1}$ Mathematical Institute, University of Oxford, \\ 24-26 St Giles, Oxford OX1 3LB,UK \\ ${ }^{2}$ Department of Mathematics, University of Utah, Salt Lake City, UT 84112
}

(Dated: August 31, 2011)

\begin{abstract}
We use the theory of noise-induced phase synchronization to analyze the effects of demographic noise on the synchronization of a metapopulation of predator-prey systems within a fluctuating environment (Moran effect). Treating each local predator-prey population as a stochastic urn model, we derive a Langevin equation for the stochastic dynamics of the metapopulation. Assuming each local population acts as a limit cycle oscillator in the deterministic limit, we use phase reduction and averaging methods to derive the steady state probability density for pairwise phase differences between oscillators, which is then used to determine the degree of synchronization of the metapopulation.
\end{abstract}

PACS numbers: 87.23.Cc, 02.50.Ey, 05.40.2a 
A major problem in ecology is understanding the mechanisms for synchronizing spatially separated populations or patches (metapopulations) [1]. It is important in conservation because synchrony is strongly correlated with the chances of global extinction [2]. On the other hand, synchrony may be desirable in the case of pest or pathogen control, since it can help to eliminate an outbreak [3]. There are two basic mechanisms for synchronizing patches within a metapopulation: isolated patches can be driven by the same environmental fluctuations (the so-called Moran effect [4]) or patches can interact with each other through dispersal in a constant environment [5-7]. The dominant mechanism will depend on the spatial scale of the metapopulation and the nature of the local patch dynamics.

In this Letter, we use the theory of noise-induced phase synchronization to analyze the effects of demographic noise on the synchronization of a metapopulation of predator-prey systems within a fluctuating environment. Noise-induced phase synchronization concerns the general dynamical theory of how a global extrinsic noise source can synchronize an ensemble of uncoupled limit cycle oscillators $[10,11]$. Although the theory has applications to a wide range of systems in the physical and life sciences (most recently neuroscience), it has not previously been applied within ecology, even though the Moran effect has been known for a long time. Moreover, in most previous applications, all sources of noise are extrinsic to the oscillator rather than partially arising from finite size effects; (see [12] for an analysis of demographic noise in neural population oscillators). We start by incorporating demographic noise into a single predator-prey system using a stochastic urn model [8]. Approximating the associated master equation using a Kramers-Moyal expansion [9], we derive a Langevin equation for an ensemble of predator-prey systems. We show that the multiplicative Gaussian noise terms can be decomposed into a set of independent white noise processes that are uncorrelated across populations (demographic noise) and an additional white noise term that is common to all populations (environmental noise). Assuming that each predator-prey system acts as a limit cycle oscillator in the deterministic limit, we use phase reduction and averaging methods to derive the steady state probability density for pairwise phase differences between oscillators, which is then used to determine the degree of synchronization of the metapopulation. We illustrate this using the Holling-Tanner model $[16,17]$.

Suppose that at time $t$ there are $m$ individuals of species $C_{1}$ (prey) and $n$ individuals of species $C_{2}$ (predators). Let the total number of individuals within a patch have an 
upper bound $N$ and use the symbol $E$ to denote the set of $N-m-n$ available slots for reproduction and intraspecies competition. Following Ref. [8], we consider a general stochastic urn model of a predator-prey system consisting of the following processes: birth processes $C_{j} E \stackrel{b_{j}}{\longrightarrow} C_{j} C_{j}$, death processes $C_{j} \stackrel{d_{j}}{\longrightarrow} E$, intraspecies competition $C_{j} C_{j} \stackrel{c_{j}}{\longrightarrow} C_{j} E$, and predator-prey interactions $C_{1} C_{2} \stackrel{p_{1}}{\longrightarrow} C_{2} E, C_{1} C_{2} \stackrel{p_{2}}{\longrightarrow} C_{2} C_{2}$. Here $b_{j}, d_{j}, c_{j}$ are the birth, death and competition rates, $p_{1}$ is the rate of prey consumption due to predation, and $p_{2}$ is the growth rate of predators at the expense of prey. Suppose that a pair of constituents is drawn with probability $\mu$ and a single constituent is drawn with probability $1-\mu$. We can then write down the following transition rates $T_{r, s}(m, n)$ from state $(m, n)$ to $(m+r, n+s)$ [8]:

$$
\begin{aligned}
T_{-1,0}(m, n) & =(1-\mu) d_{1} m / N+2 \mu c_{1} \eta_{m, m-1}+2 \mu p_{1} \eta_{m, n} \\
T_{0,-1}(m, n) & =(1-\mu) d_{2} n / N+2 \mu c_{2} \eta_{n, n-1} \\
T_{1,0}(m, n) & =2 \mu b_{1} \eta_{m, N-m-n} \\
T_{0,1}(m, n) & =2 \mu b_{2} \eta_{n, N-m-n}, T_{-1,1}(m, n)=2 \mu p_{2} \eta_{m, n} .
\end{aligned}
$$

where $\eta_{n, m}=n m /(N(N-1))$. The master equation for the probability $\mathbb{P}_{m, n}(t)$ that the system consists of $m$ prey and $n$ predators at time $t$ then takes the form

$$
\frac{d \mathbb{P}_{m, n}}{d t}=\sum_{r, s}\left[T_{r, s}\left(m^{\prime}, n^{\prime}\right) \mathbb{P}_{m^{\prime}, n^{\prime}}-T_{r, s}(m, n) \mathbb{P}_{m, n}\right]
$$

with $m^{\prime}=m-r, n^{\prime}=n-s$.

An exact solution of the master equation (1) can rarely be found, so it is necessary to consider some form of approximation. In particular, for large $N$ we can carry out a Kramers-Moyal expansion of the master equation (1) [9]. Introduce new variables $x_{1}=$ $m / N, x_{2}=n / N$ and set $\mathbb{P}_{m, n}(t)=\mathbb{P}\left(x_{1}, x_{2}, t\right)$. Treating $x_{1}, x_{2}$ as continuous variables and Taylor expanding each term on the right-hand side of equation (1) to second order in $1 / N$ yields the Fokker-Planck (FP) equation

$$
\frac{\partial \mathbb{P}}{\partial t}=-\sum_{i=1,2} \frac{\partial\left(A_{i}(\mathbf{x}) \mathbb{P}\right)}{\partial x_{i}}+\frac{\epsilon^{2}}{2} \sum_{i, j=1,2} \frac{\partial^{2}\left(G_{i j}(\mathbf{x}) \mathbb{P}\right)}{\partial x_{i} \partial x_{j}}
$$

where $\mathbf{x}=\left(x_{1}, x_{2}\right), \epsilon=1 / \sqrt{N} \ll 1$,

$$
\begin{aligned}
A_{1}(\mathbf{x})= & 2 b_{1} x_{1}\left(1-x_{1}-x_{2}\right)-2 c_{1} x_{1}^{2}-d_{1} x_{1} \\
& -2\left(p_{1}+p_{2}\right) x_{1} x_{2} \\
A_{2}(\mathbf{x})= & 2 b_{2} x_{2}\left(1-x_{1}-x_{2}\right)-2 c_{2} x_{2}^{2}-d_{2} x_{2}+2 p_{2} x_{1} x_{2}
\end{aligned}
$$


and $G_{j j}(\mathbf{x})$ is obtained from $A_{j}(\mathbf{x})$ by reversing the minus sign on the last three terms, whereas $G_{12}(\mathbf{x})=G_{21}(\mathbf{x})=-2 p_{2} x_{1} x_{2}$. We have rescaled the various rates according to $d_{k} \rightarrow(1-\mu) d_{k} / N, b_{k} \rightarrow \mu b_{k} /(N-1), c_{k} \rightarrow \mu c_{k} /(N-1), p_{k} \rightarrow \mu p_{k} /(N-1)$.

Next we introduce the three stoichiometric vectors $\mathbf{v}_{1}=(1,0)^{T}, \mathbf{v}_{2}=(0,1)^{T}, \mathbf{v}_{3}=$ $(-1,1)^{T}$, which (up to a sign) represent all possible increments in the number of predator and prey induced by single interactions. For example, predation $C_{1} C_{2} \rightarrow C_{2} C_{2}$ is represented by the stoichiometric vector $(-1,1)^{T}$. It can then be shown that the solution to the FP equation (2) determines the probability density function for a corresponding stochastic process $\mathbf{X}=\left(X_{1}, X_{2}\right)$ that evolves according to an Itō type Langevin equation $d \mathbf{X}=\mathbf{A}(\mathbf{X}) d t+\epsilon \sum_{i=1}^{3} \mathbf{B}^{(i)}(\mathbf{X}) d W_{i}$, where $\mathbf{B}^{(j)}=\sqrt{G_{j j}(\mathbf{x})-2 p_{2} x_{1} x_{2}} \mathbf{v}_{j}, j=1,2$, and $\mathbf{B}^{(3)}=\sqrt{2 p_{2} x_{1} x_{2}} \mathbf{v}_{3}$. Here $W_{i}$ denotes an independent Wiener process such that $\left\langle d W_{i}(t)\right\rangle=0$ and $\left\langle d W_{i}(t) d W_{j}(t)\right\rangle=\delta_{i, j} d t$. The Langevin equation approximates the effects of demographic noise arising from the finite size $N$ of the local predator-prey population. It is also possible to include a common extrinsic noise source due to environmental fluctuations. For concreteness, suppose that we add a stochastic term to the birth rates $b_{k}$ according to $b_{k} \rightarrow b_{k}+\sigma \xi(t) / 2$, where $\xi(t)$ is a white noise term and $\sigma$ is the strength of environmental noise. Substituting for $b_{k}$ in equation (3) and expanding to $\mathcal{O}(\sigma)$ leads to an additional multiplicative term in the Langevin equation of the form $\sigma \mathbf{H}(\mathbf{X}) d W$, where $\mathbf{H}=\left(H_{1}, H_{2}\right)$ with $H_{1}(\mathbf{X})=r_{1} x_{1}\left(1-x_{1}-x_{2}\right), H_{2}(\mathbf{X})=r_{2} x_{2}\left(1-x_{1}-x_{2}\right)$, and $d W(t)=\xi(t) d t$ is an additional independent Wiener process, which is treated in the sense of Stratonovich.

In the deterministic limit $(\epsilon, \sigma \rightarrow 0)$ the Langevin equation reduces to the deterministic planar dynamical system $\dot{x}_{1}=A_{1}(\mathbf{x}), \dot{x}_{2}=A_{2}(\mathbf{x})$ with $A_{j}$ given by equation (3). Suppose that the deterministic system supports a limit cycle solution $\mathbf{x}=\mathbf{x}^{*}(t)$ with $\mathbf{x}^{*}(t+k T)=$ $\mathbf{x}^{*}(t)$ for all integers $k$, where $T$ is the period of oscillations. Now consider an ensemble of $\mathcal{N}$ identical, spatially separated predator-prey patches, each of which acts as a limit cycle oscillator in the deterministic limit. If we ignore spatial interactions (dispersal, migration) between the isolated patches, then we have a system of uncoupled population oscillators driven by a common environmental noise source. Introducing the label $\mu, \mu=1, \ldots, \mathcal{N}$, the Langevin equation for the ensemble of patches is

$$
d \mathbf{X}^{(\mu)}=\mathbf{A}^{(\mu)} d t+\epsilon \sum_{i=1}^{3} \mathbf{B}^{(i, \mu)} d W_{i}^{(\mu)}+\sigma \mathbf{H}^{(\mu)} d W,
$$

where $\mathbf{A}^{(\mu)}=\mathbf{A}\left(\mathbf{X}^{(\mu)}\right)$ etc. We associate an independent set of Wiener processes $W_{j}^{(\mu)}$ 
with each population oscillator (demographic noise) but take the environmental noise to be common to all the oscillators: $\left\langle d W_{i}^{(\mu)}(t) d W_{j}^{(\nu)}(t)\right\rangle=\delta_{i, j} \delta_{\mu, \nu} d t,\left\langle d W_{i}^{(\mu)}(t) d W(t)\right\rangle=0$, $\langle d W(t) d W(t)\rangle=d t$.

Following previous studies of noise-induced phase synchronization [10-12], we now carry out a stochastic phase reduction of the Langevin equation (4). First, we introduce the phase variable $\theta \in(-\pi, \pi]$ such that the dynamics of a single oscillator in the absence of noise reduces to the simple phase equation $\dot{\theta}=\omega_{0}$, where $\omega_{0}=2 \pi / T$. We then extend the notion of phase into some neighbourhood $\mathcal{M} \subset \mathbb{R}^{2}$ of the limit cycle using an isochronal mapping $\Pi: \mathcal{M} \rightarrow(-\pi, \pi]$, with $\theta=\Pi(\mathbf{x})$, assuming that the limit cycle is sufficiently attracting so that for small $\epsilon$ and $\sigma$, the dynamics can be restricted to the neighborhood $\mathcal{M}$ with high probability [13]. This allows us to define a stochastic phase variable for each oscillator according to $\Theta^{(\mu)}=\Pi\left(\mathbf{X}^{(\mu)}\right)$ with $\mathbf{X}^{(\mu)}$ evolving according to the Langevin equation (4). After converting the demographic noise terms in equation (4) to Stratonovich form (which adds an $\mathcal{O}\left(\epsilon^{2}\right)$ correction to the drift terms [12]), we perform a change of variables $\mathbf{X}^{(\mu)} \rightarrow\left(\Theta^{(\mu)}, r^{(\mu)}\right)$ and then project out the phase dynamics along the lines of Ref. [14]. This ultimately leads to an Ito Langevin equation for the stochastic phase variables $\Theta^{(\mu)}$ of the form

$$
d \Theta^{(\mu)}=\omega^{(\mu)} d t+\epsilon \sum_{i=1}^{3} \beta_{i}^{(\mu)} d W_{i}^{(\mu)}+\sigma \alpha^{(\mu)} d W
$$

with $\beta_{i}^{(\mu)}=\beta_{i}\left(\Theta^{(\mu)}\right) \equiv \mathbf{Z}\left(\Theta^{(\mu)}\right) \cdot \mathbf{B}^{(i)}\left(\Theta^{(\mu)}\right), \alpha^{(\mu)}=\alpha\left(\Theta^{(\mu)}\right) \equiv \mathbf{Z}\left(\Theta^{(\mu)}\right) \cdot \mathbf{H}\left(\Theta^{(\mu)}\right)$ and $\omega^{(\mu)}=$ $\omega\left(\Theta^{(\mu)}\right) \equiv \omega_{0}+\epsilon^{2} \Omega_{1}\left(\Theta^{(\mu)}\right)+\sigma^{2} \Omega_{2}\left(\Theta^{(\mu)}\right)$. Here $\mathbf{Z}=\left(Z_{1}, Z_{2}\right)$ where $Z_{k}, k=1,2$, is the $k$ th component of the infinitesimal phase resetting curve (PRC), which is defined as $Z_{k}(\theta) \equiv$ $\left.\partial_{x_{k}} \Pi\right|_{\mathbf{x}=\mathbf{x}^{*}(\theta)}$ with $\sum_{k=1,2} Z_{k}(\theta) A_{k}\left(\mathbf{x}^{*}(\theta)\right)=\omega$. The PRC is the unique, normalized $2 \pi-$ periodic solution of the adjoint equation [18] $\dot{Z}_{k}=-\sum_{j=1,2} A_{j k}\left(\mathbf{x}^{*}(t)\right) Z_{j}(t)$, where $A_{j k}=$ $\partial A_{j} / \partial x_{k}$. All terms in equation (5) are evaluated on the limit cycle so that, for example, $\mathbf{B}^{(i)}(\theta)=\mathbf{B}^{(i)}\left(\mathbf{x}^{*}(\theta)\right)$. As first shown in Ref. [14], and further developed in Refs. [15], considerable care must be taken in carrying out the phase reduction procedure in the presence of Gaussian white noise in order to obtain the correct form of the drift terms $\Omega_{1}, \Omega_{2}$. However, the latter will not be important in the following analysis.

Following Ref. [11], we now introduce slow phase variables $\psi^{(\mu)}=\Theta^{(\mu)}-\omega_{0} t$ and set $Q\left(\left\{\psi^{(\mu)}\right\}, t\right)=P\left(\left\{\psi^{(\mu)}-\omega_{0} t\right\}, t\right)$ where $P$ is the probability density of the phases $\Theta^{(\mu)}$. For small $\sigma$ and $\epsilon, Q$ is a slowly varying function of time, so we can av- 
erage the FP equation for $Q$ over one cycle of length $T=2 \pi / \omega_{0}$ to get $\partial_{t} Q=$ $-\bar{\Omega} \sum_{\mu=1}^{\mathcal{N}} \partial_{\psi^{(\mu)}} Q+\frac{1}{2} \sum_{\mu=1}^{\mathcal{N}} \sum_{\nu=1}^{\mathcal{N}} \partial_{\psi^{(\mu)} \psi^{(\nu)}}\left[\bar{C}^{(\mu \nu)} Q\right]$, where $\bar{\Omega}=\frac{1}{2 \pi} \int_{-\pi}^{\pi}\left[\epsilon^{2} \Omega_{1}(\theta)+\sigma^{2} \Omega_{2}(\theta] d \theta\right.$, $\bar{C}^{(\mu \nu)}=\sigma^{2} g\left(\psi^{(\mu)}-\psi^{(\nu)}\right)+\epsilon^{2} h(0) \delta_{\mu, \nu}$ with $g(\psi)=\frac{1}{2 \pi} \int_{-\pi}^{\pi} \alpha\left(\theta^{\prime}\right) \alpha\left(\theta^{\prime}+\psi\right) d \theta^{\prime}$ and $h(\psi)=$ $\frac{1}{2 \pi} \int_{-\pi}^{\pi} \sum_{i=1}^{3} \beta_{i}\left(\theta^{\prime}\right) \beta_{i}\left(\theta^{\prime}+\psi\right) d \theta^{\prime}$. In order to characterize the level of synchrony, we focus on a pair of population oscillators $(\mathcal{N}=2)$. Performing the change of variables $\psi=\left(\psi^{(1)}+\psi^{(2)}\right) / 2, \phi=\psi^{(1)}-\psi^{(2)}$, and looking for separable steady-state solutions $Q\left(\psi^{(1)}, \psi^{(2)}\right)=\Psi(\psi) \Phi(\phi)$, it can be shown that $[11]$

$$
\Phi(\phi)=\Gamma_{0}\left[\sigma^{2}(g(0)-g(\phi))+\epsilon^{2} h(0)\right]^{-1}
$$

with $\Gamma_{0}$ a normalization constant, and $\Psi(\psi)=1 / 2 \pi$. We see that $\Phi(\phi)$ is independent of the drift terms $\Omega_{j}(\theta)$.

In the thermodynamic limit $N \rightarrow \infty$ we have $\epsilon=N^{-1 / 2} \rightarrow 0$ so that the independent noise source vanishes. The distribution $\Phi(\phi)$ then diverges at $\phi=0$ while keeping positive since it can be shown that $g(0) \geq g(\theta)[11]$. Hence, the phase difference between any pair of oscillators accumulates at zero, resulting in complete noise-induced synchronization. Thus, a randomly fluctuating global environmental signal can synchronize a metacommunity of deterministic predator-prey systems in the absence of dispersal, consistent with the Moran effect [4]. However, for finite $N$, demographic noise broadens the distribution of phase differences, resulting in the desynchronization of the metacommunity. As we now show, the width of $\Phi(\phi)$ is a good measure of the desynchronizing effects of demographic noise.

As an illustrative example of an oscillatory predator-prey system, consider the HollingTanner model $[16,17]$

$$
\dot{x}_{1}=r x_{1}\left(1-\frac{x_{1}}{K}\right)-\frac{m x_{1} x_{2}}{A_{0}+x_{1}}, \dot{x}_{2}=s x_{2}\left(1-\frac{h x_{2}}{x_{1}}\right) .
$$

In this model, the prey exhibit logistic growth up to a carrying capacity $K$ in the absence of predation. The predator consumes prey according to a Michaelis-Menten or Holling type-II functional response and grows logistically up to a carrying capacity $x / h$ proportional to the current level of prey in the system. It is straightforward to find a set of parameters for which equation (7) supports a limit cycle [17] and to numerically evaluate the corresponding PRCs, see Fig. 1(a). Comparing the right-hand side of equations (7) with the drift functions of equation (3), we set $b_{1}=r / 2 K, b_{2}=\left(s+d_{2}\right) / 2, d_{1}=r(1 / K-1), c_{1}=0, c_{2}=$ $s h / 2 x_{1}-\left(s+d_{2}\right) / 2, p_{1}=m /\left[2\left(A_{0}+x_{1}\right)\right]-\left(s+d_{2}\right) / 2-r / 2 K, p_{2}=\left(s+d_{2}\right) / 2$, where $d_{2}$ is 
a free parameter. For concreteness, we set $d_{2}=s$. We can now determine the functions $g, h$ in equation (6) for an ensemble of Holling-Tanner oscillators using the given PRC, see Fig. $1(\mathrm{~b})$.
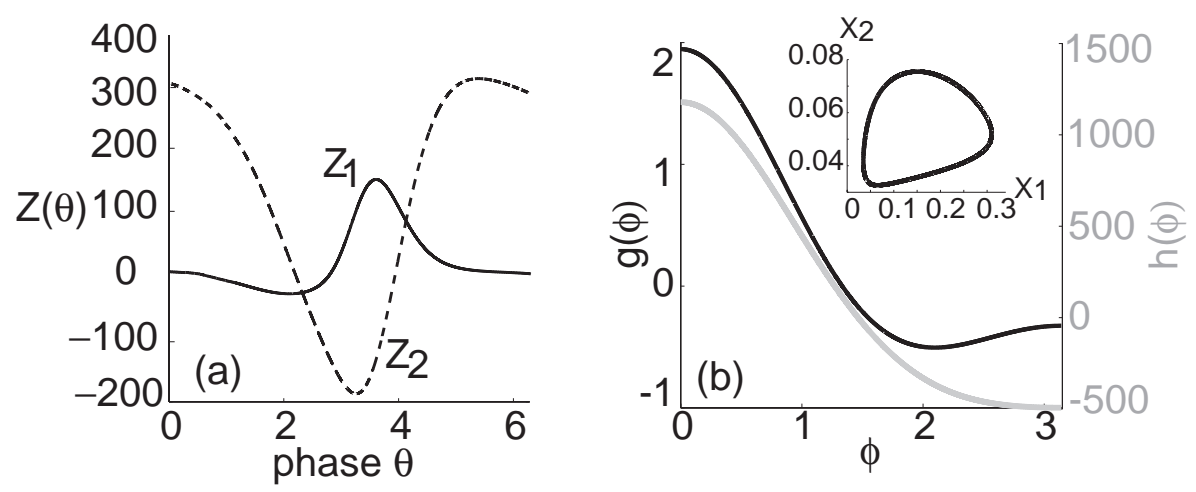

FIG. 1: (a) Plot of components $Z_{1}, Z_{2}$ of PRC for a limit cycle solution of the Holling-Tanner equations with $r=1, A_{0}=0.1, s=0.1, h=2, m=3$ and $K=0.5$. (b) Corresponding functions $g(\phi)$ and $h(\phi)$. Inset shows limit cycle.

In Fig. 2 we relate our analytical results for the distribution $\Phi(\phi)$ to direct numerical simulations of the Langevin equation (4) for the Holling-Tanner model. The simulations are carried out using an Euler-Maruyama scheme, with the Stratonovich environmental noise treated as a solution of a zero-mean Ornstein-Uhlenbeck process with a small but finite correlation time. In order to quantify the effects of demographic noise on the metapopulation, we introduce ensemble averaged concentrations of prey and predator according to $\bar{X}_{j}(t)=\mathcal{N}^{-1} \sum_{\mu=1}^{\mathcal{N}} X_{j}^{(\mu)}(t), \quad j=1,2$. For a high ratio of extrinsic to intrinsic noise, there is significant synchronization of the metapopulation, as indicated by the sharp peak of the steady-state distribution $\Phi(\phi)$ at $\phi=0$ in Fig. 2(a) and the corresponding clustering of the individual oscillators on the deterministic limit cycle (Fig. 2(d)). It can also be seen that $\bar{X}_{j}(t)$ traces out a trajectory in the phase plane that remains close to the deterministic limit cycle. For a moderate ratio of extrinsic to intrinsic noise, the metapopulation is only partially synchronized. The distribution $\Phi$ is now much broader (Fig. 2(b)) and, although the oscillators tend to remain close to the deterministic limit cycle, they are no longer tightly clustered. Thus, $\bar{X}_{j}(t)$ still exhibits some oscillatory behavior but the amplitude of oscillations is reduced (Fig.2(e)). Finally, for a low ratio of extrinsic to intrinsic noise, the metapopulation is completely desynchronized. The density $\Phi$ is almost flat (Fig. 

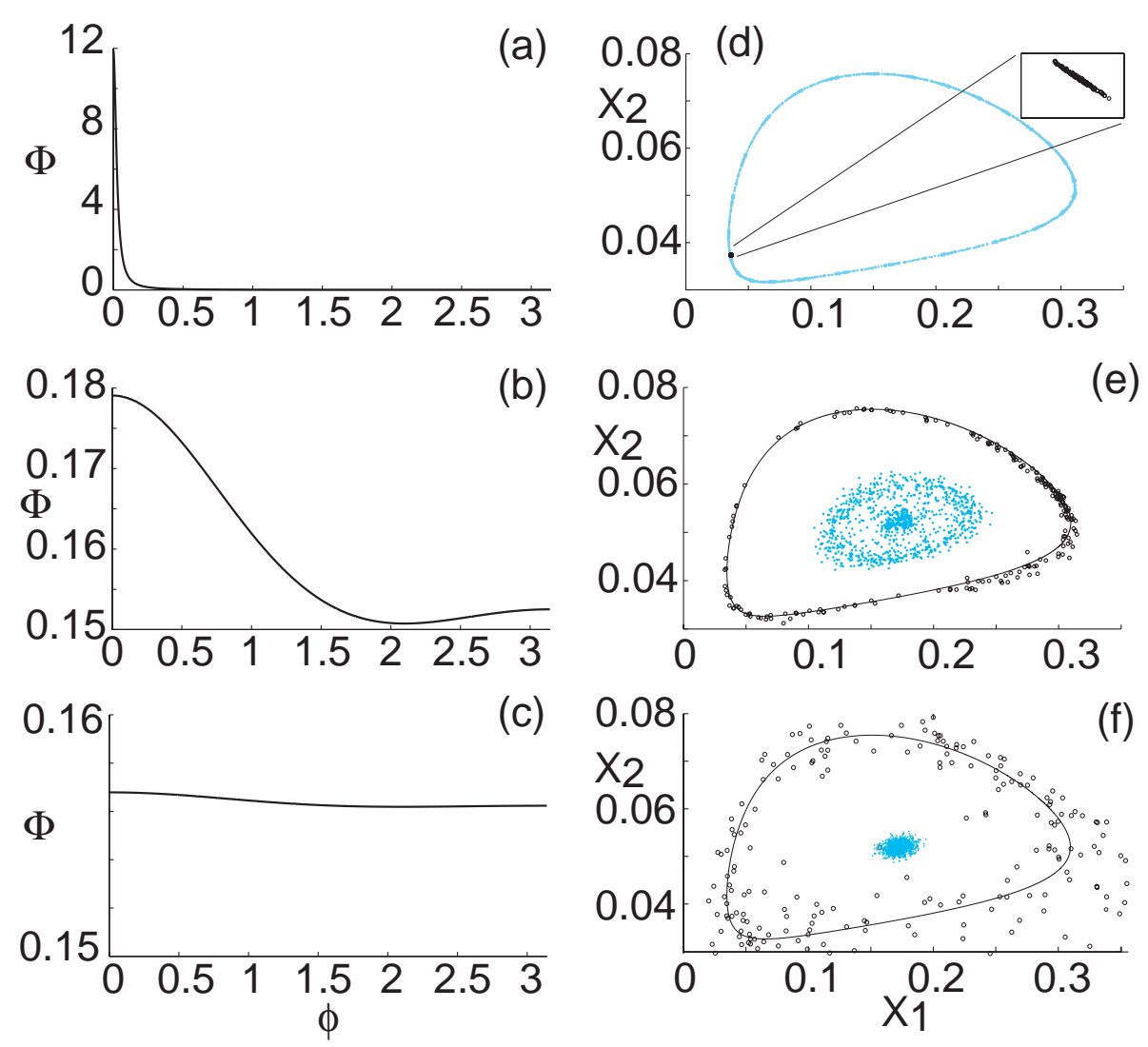

FIG. 2: Comparison of numerical simulations and analytical results for $\mathcal{N}=200$ Holling-Tanner oscillators using same parameters as Fig. 1. (a)-(c) Steady-state probability density $\Phi(\phi)$ of pairwise phase differences for various levels of environmental and demographic noise: a) $\sigma=10^{-3}$, $\epsilon=10^{-6}$ b) $\sigma=10^{-2}$ and $\epsilon=10^{-3}$ c) $\sigma=10^{-6}$ and $\epsilon=10^{-3}$. (d)-(f). Corresponding ensemble dynamics in state space showing a snapshot of all the oscillators (black dots) and a scatterplot of the averaged trajectory $\bar{X}_{j}(t)$ of the metapopulation over multiple realizations (blue/gray dots).

$2(\mathrm{c}))$, and individual oscillators are much more scattered in the phase-plane so that $\bar{X}_{j}(t)$ exhibits small fluctuations about the mean of the limit cycle (Fig. 2(f)). In order to further quantify how demographic noise affects synchrony, we introduce the order parameter $R(t)=\mathcal{N}^{-1}\left|\sum_{\mu=1}^{\mathcal{N}} \mathrm{e}^{i \Theta^{(\mu)}(t)}\right|$ and the half-width $\Delta$ with $\int_{-\Delta / 2}^{\Delta / 2} \Phi(\phi) d \phi=0.5$. In Fig. 3 we show how the half-width $\Delta$ provides an excellent predictor of the level of synchrony by comparing its dependence on system size $N$ with the time average of $R(t)$.

In conclusion, we have applied stochastic phase reduction and averaging methods to analzye an important ecological problem, namely, the effects of demographic noise on the synchronization of a metapopulation in a fluctuating environment (Moran effect). We have 


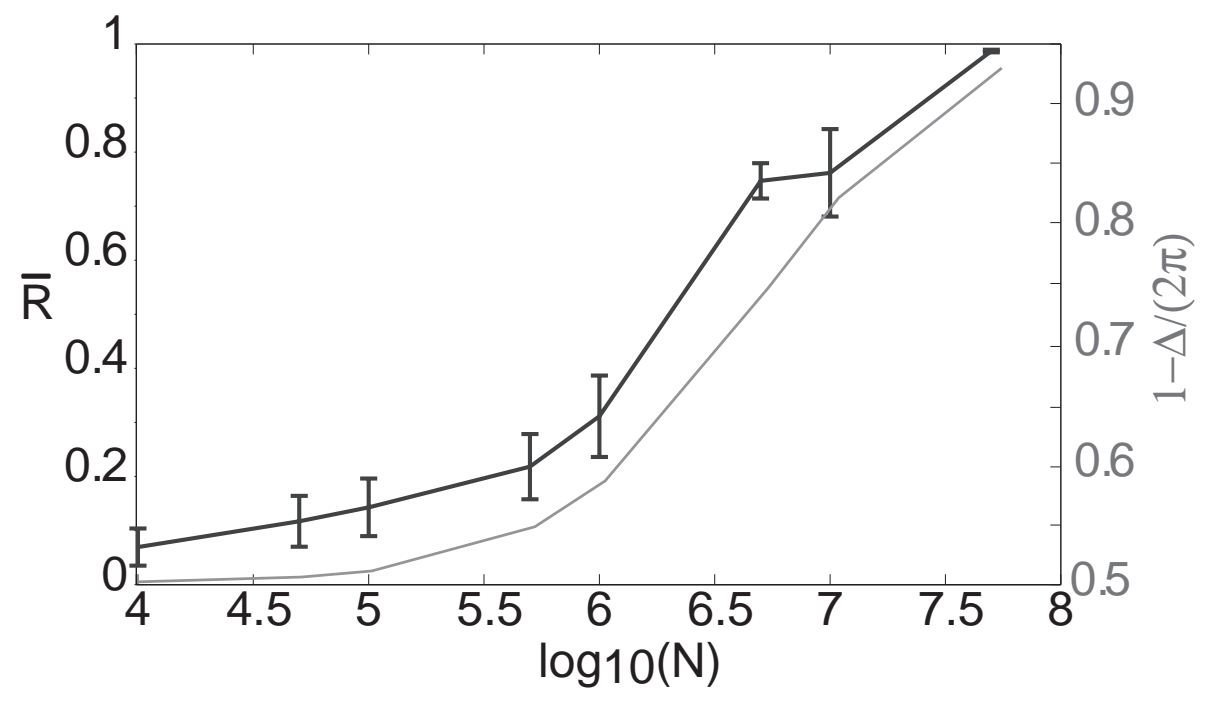

FIG. 3: Plots of time-averaged order parameter $\bar{R}$ (black curve) and half-width $1-\Delta /(2 \pi)$ (gray curve) as a function of system size $N$. Variance of $R(t)$ with respect to time is shown by error bars. Parameters as in Fig. 2 with $\sigma=0.01$.

shown that the degree of synchronization of the metapopulation can be characterized in terms of the probability density for pairwise phase differences. One major implication of our work is that the stabilizing effects of demographic noise (Fig. 2(f)) could provide an explanation for why oscillations are often not observed in real ecological systems, contrary to predictions from deterministic models [19]. There are numerous ecologically motivated extensions of this work, including dispersal between local patches, spatial heterogeneity [20], and the decay of synchrony with spatial separation of the patches $[1,2]$.

Acknowledgments: This work was partly supported by King Abdullah University of Science and Technology Award No. KUK-C1-013-04.

[1] P. J. Hudson and I. M. Cattadori. Trends Ecol. Evol. 141 (1999).

[2] I. Hanski. Nature 396, 42 (1998).

[3] D. J. D. Earn, P. Rohani and B. T. Grenfell. Proc. Roy. Soc. B 265, 7 (1998); P. Rohani, D. J. D. Earn and B. T. Grenfell. Science 286968 (1999).

[4] P. A. P. Moran. Austral. J. Zoology. 1, 291 (1953).

[5] L. M. Pecora and T. L. Carroll. Phys. Rev. Lett. 80, 2109 (1998). 
[6] V. A. A. Jansen and A. L. Lloyd. J. Math. Biol. 41, 232 (2000).

[7] D. A. Vasseur and J. W. Fox. Nature, 460, 1007 (2009).

[8] A.J. McKane and T.J. Newman, Phys. Rev. E 70, 041902 (2004); Phys. Rev. Lett. 94, 218102 (2005).

[9] C.W. Gardiner, Stochastic methods: a handbook for the natural and social sciences. 4th ed. (Springer, NY, 2009).

[10] A. S. Pikovsky Radiophys., 27:576-581 (1984);J. N. Teramae and D. Tanaka Phys. Rev. Lett. 93:204103 (2004); D. S. Goldobin and A. Pikovsky. Phys. Rev. E, 71:045201 (2005); C. Ly and G. B. Ermentrout. J. Comput. Neurosci., 26:425-443 (2009).

[11] H. Nakao, K. Arai, and Y. Kawamura, Phys. Rev. Lett., 98, 184101 (2007).

[12] P. C. Bressloff and Y.M. Lai. J. Math. Neurosci. 1,2 (2011).

[13] In the case of Gaussian noise there is a small but nonzero probability for large deviations under which the phase reduction method could break down. However, this does not affect the theoretical arguments presented here.

[14] K. Yoshimura and K. Arai. Phys. Rev. Lett. 101154101 (2008).

[15] J. N. Teramae, H. Nakao and G. B. Ermentrout. Phys. Rev. Lett., 102:194102 (2009); Goldobin et. al. Phys. Rev. Lett. 105154101 (2010); K. Yoshimura. In Reviews in nonlinear dynamics and complexity vol. 3, H. G. Schuster (ed). Ch. 3 (Wiley, 2010).

[16] J. T. Tanner. Ecology 56, 855 (1975).

[17] A. Gasull, R.E. Kooij, and J. Torregrosa, Publicacions Matematiques, 41 (1997), 149-167.

[18] G. B. Ermentrout and N. Kopell. J. Math. Biol. 29:195-217 (1991).

[19] W. W. Murdoch, R. M. Nisbet, E. McCauley, A. M. deRoos and W. S. C. Gurney. Ecology 791339 (1998).

[20] K. Yoshimura et al, Progress of Theoretical Physics 120, 621 (2008) 



\section{RECENT REPORTS}

24/11 On approaches to modelling lattice dislocations Hall

Markenscoff

25/11 Nonlinear waves in heterogeneous elastic rods via homogenization de Luna

Emptage

Goriely

Bressloff

26/11 Synaptic bistability due to nucleation and evaporation of receptor clusters

Burlakov

Duričković

Goriely

27/11 Particle trapping and banding in rapid solidification

Elliot

Peppin

28/11 Growth of confined cancer spheroids: a combined experimental and mathematical modelling approach

Loessner

Flegg

Byrne

Hall

Moroney

Clements

McElwain

Hutmacher

29/11 Floating carpets and the delamination of elastic sheets Wagner

Vella

30/11 Numerical Study of Liquid Crystal Elastomers by a Mixed Finite

Luo

Calderer

31/11 The indentation of pressurized elastic shells: From polymeric cap-

Vella sules to yeast cells

Ajdari

Vaziri

Boudaoud

32/11 Wrinkling of pressurized elastic shells

Vella

Ajdari

Vaziri

Boudaoud

33/11 Data assimilation using bayesian filters and B-spline geological models

Duan

Farmer

Hoteit

$\mathrm{Lu}$

Moroz

34/11 Review of nonlinear Kalman, ensemble and particle filtering with application to the reservoir history matching problem

Luo

Hoteit

Duan

Wang

35/11 Modelling a Tethered Mammalian Sperm Cell undergoing Hyperactivation

Curtis

Kirkman-Brown

Connolly

Gaffney

36/11 A simple mathematical model for investigating the effect of cluster

Zygalakis roots on plant nutrient uptake

Roose 
40/11 Higher-order numerical methods for stochastic simulation of Székely chemical reaction systems

Burrage

Erban

Zygalakis

41/11 On the modelling and simulation of a high pressure shift freezing

Smith process

Peppin

Ángel M. Ramos

42/11 An efficient implementation of an implicit FEM scheme for

Burrage fractional-in-space reaction-diffusion equations

Hale

Kay

43/11 Coupling fluid and solute dynamics within the ocular surface tear film: a modelling study of black Line osmolarity

Zubkov

Breward

Gaffney

44/11 A prototypical model for tensional wrinkling in thin sheets

Davidovitch

Schroll

Vella

Adda-Bedia

Cerda

45/11 A fibrocontractive mechanochemical model of dermal wound closure incorporating realistic growth factor

Murphy

Hall

Maini

$\mathrm{McCue}$

McElwain

46/11 A two-compartment mechanochemical model of the roles of trans-

Murphy forming growth factor $\beta$ and tissue tension in dermal wound healing

Hall

Maini

$\mathrm{McCue}$

McElwain

Copies of these, and any other OCCAM reports can be obtained from:

Oxford Centre for Collaborative Applied Mathematics

Mathematical Institute

24 - 29 St Giles'

Oxford

OX1 3LB

England

www.maths.ox.ac.uk/occam 